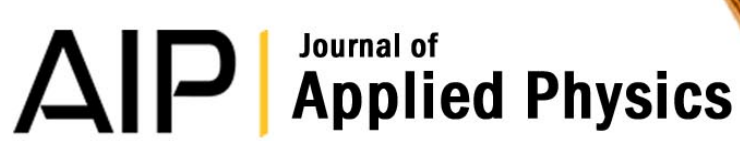

Piezoresponse force microscopy studies on the domain structures and local switching behavior of $\mathrm{Pb}(\ln 1 / 2 \mathrm{Nb} 1 / 2) \mathrm{O} 3-\mathrm{Pb}(\mathrm{Mg} 1 / 3 \mathrm{Nb} 2 / 3) \mathrm{O} 3-\mathrm{PbTiO} 3$ single crystals

Qian Li, Yun Liu, Ray L. Withers, Yuhui Wan, Zhenrong Li et al.

Citation: J. Appl. Phys. 112, 052006 (2012); doi: 10.1063/1.4745979

View online: http://dx.doi.org/10.1063/1.4745979

View Table of Contents: http://jap.aip.org/resource/1/JAPIAU/v112/i5

Published by the American Institute of Physics.

\section{Related Articles}

Influence of target composition and deposition temperature on the domain structure of $\mathrm{BiFeO} 3$ thin films AIP Advances 2, 042104 (2012)

Nanodomain structures formation during polarization reversal in uniform electric field in strontium barium niobate single crystals

J. Appl. Phys. 112, 064117 (2012)

The effect of the top electrode interface on the hysteretic behavior of epitaxial ferroelectric $\mathrm{Pb}(\mathrm{Zr}, \mathrm{Ti}) \mathrm{O} 3$ thin films with bottom $\mathrm{SrRuO} 3$ electrode

J. Appl. Phys. 112, 064116 (2012)

Ferroelectric vs. structural properties of large-distance sputtered epitaxial LSMO/PZT heterostructures AlP Advances 2, 032184 (2012)

Piezoelectric nonlinearity and frequency dispersion of the direct piezoelectric response of $\mathrm{BiFeO} 3$ ceramics J. Appl. Phys. 112, 064114 (2012)

\section{Additional information on J. Appl. Phys.}

Journal Homepage: http://jap.aip.org/

Journal Information: http://jap.aip.org/about/about_the_journal

Top downloads: http://jap.aip.org/features/most_downloaded

Information for Authors: http://jap.aip.org/authors

\section{ADVERTISEMENT}

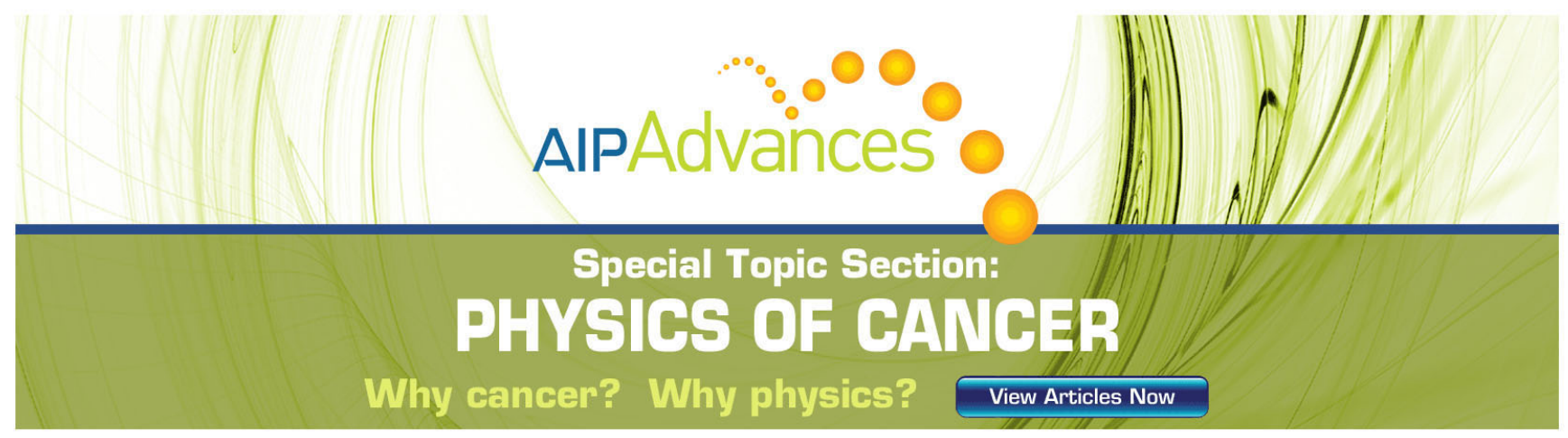




\title{
Piezoresponse force microscopy studies on the domain structures and local switching behavior of $\mathrm{Pb}\left(\ln _{1 / 2} \mathrm{Nb}_{1 / 2}\right) \mathrm{O}_{3}-\mathrm{Pb}\left(\mathrm{Mg}_{1 / 3} \mathrm{Nb}_{2 / 3}\right) \mathrm{O}_{3}-\mathrm{PbTiO}_{3}$ single crystals
}

\author{
Qian Li, ${ }^{1}$ Yun Liu, ${ }^{1, a)}$ Ray L. Withers, ${ }^{1}$ Yuhui Wan, ${ }^{2}$ Zhenrong $\mathrm{Li},{ }^{2}$ and Zhuo $\mathrm{Xu}^{2}$ \\ ${ }^{1}$ Research School of Chemistry, The Australian National University, ACT 0200, Australia \\ ${ }^{2}$ Electronic Materials Research Laboratory (EMRL), Xi' an Jiaotong University, Shaanxi 710049, China
}

(Received 4 December 2011; accepted 18 April 2012; published online 4 September 2012)

\begin{abstract}
The static domain structures and local switching behavior of relaxor ferroelectric $0.29 \mathrm{~Pb}\left(\operatorname{In}_{1 / 2} \mathrm{Nb}_{1 / 2}\right)$ $\mathrm{O}_{3}-0.44 \mathrm{~Pb}\left(\mathrm{Mg}_{1 / 3} \mathrm{Nb}_{2 / 3}\right) \mathrm{O}_{3}-0.27 \mathrm{PbTiO}_{3}$ single crystals at three crystal orientations are studied using piezoresponse force microscopy (PFM). PFM domain imaging shows that both the [001] and [111] oriented crystals exhibit a labyrinth-like surface domain pattern while at the [110] crystal orientation the domains are preferentially aligned along $\langle 110\rangle$ directions. Vertical and lateral PFM images are used in combination to discuss the polarization vector alignment underlying the observed domain behaviour. Piezoresponse hysteresis loops acquired from these crystals indicate that the [111] crystal orientation has distinct local switching characteristics from the other two orientations in terms of, e.g., coercive voltage. Moreover, we investigate the tip-induced domain growth kinetics on an externally poled [001] crystal, the results of which exemplify an important role of extrinsic factors, e.g., the charge injection effect, in the domain patterning process. (c) 2012 American Institute of Physics. [http://dx.doi.org/10.1063/1.4745979]
\end{abstract}

\section{INTRODUCTION}

With the elucidation of the theory of imaging and concurrent developments in the instrumentation associated with atomic force microscopy (AFM), piezoresponse force microscopy (PFM) has evolved into a mature technique for the characterization of piezoelectric and ferroelectric properties on the nanoscale. ${ }^{1-4}$ PFM studies are also providing crucial insight into fundamental physical problems. A prominent example is PFM studies on the technologically important class of relaxor ferroelectric single crystals (RFSCs).

RFSCs are known to exhibit complex microstructures over several length scales, thus necessitating a combination of different microscopic methods to provide an appropriate physical picture of their nature. While diffraction-based approaches are widely employed to study the average and local structures of RFSCs, PFM can directly probe local piezoelectric and ferroelectric properties as well as image electric domains. It has been revealed by PFM, for example, that RFSCs commonly exhibit a labyrinth-like domain configuration with sizes ranging from tens to a few hundreds of nanometers (mainly observed on (001) surfaces; note that pseudocubic crystallographic indexing is used in this paper). ${ }^{5-9}$ Attempts have also been made to correlate these labyrinth-like domain configurations with structurally disordered polar nanoregions (PNRs), the existence of which is believed to be a unique characteristic of relaxor materials. ${ }^{10}$ Time-resolved PFM spectroscopic measurements suggest the existence of two distinct types of PNRs in RFSCs: static PNRs and dynamic PNRs, the former being responsible for the observed labyrinth-like domains and the latter for the relaxation behavior observed in piezoresponse $(P R)^{7,8}$

\footnotetext{
a) Author to whom correspondence should be addressed. Electronic mail: yliu@rsc.anu.edu.au.
}

PFM also provides the capacity to create and manipulate electrical domains on the surfaces of ferroelectric materials. Such artificial domain structures have much potential for use in memory devices, as nanostructure growth templates as well as for nonlinear optics applications. ${ }^{11}$ The excellent physical properties and availability of large scale, high quality crystals make RFSCs ideal candidates for these applications. Therefore, it is of considerable significance to investigate the local domain switching behavior of RFSCs.

In this paper, we investigate a ternary $\mathrm{Pb}\left(\operatorname{In}_{1 / 2} \mathrm{Nb}_{1 / 2}\right) \mathrm{O}_{3^{-}}$ $\mathrm{Pb}\left(\mathrm{Mg}_{1 / 3} \mathrm{Nb}_{2 / 3}\right) \mathrm{O}_{3}-\mathrm{PbTiO}_{3}$ (hereafter PIMNT) single crystal using PFM. The composition chosen is located close to, but slightly on the rhombohedral side of, the morphotropic phase boundary (MPB) region. The PIMNT sample used is thus a slightly rhombohedrally distorted RFSC (see supplementary material S3 for evidence of the relaxor nature of the sample). ${ }^{12}$ Our study has involved not only the domain structures that accompany the relaxor behavior of these PIMNT crystals but also their local switching behavior, with an emphasis on delineating the effects of extrinsic factors, for example, the charge injection effect.

\section{EXPERIMENT}

Near-MPB $0.29 \mathrm{~Pb}\left(\mathrm{In}_{1 / 2} \mathrm{Nb}_{1 / 2}\right) \mathrm{O}_{3^{-}}-0.44 \mathrm{~Pb}\left(\mathrm{Mg}_{1 / 3} \mathrm{Nb}_{2 / 3}\right) \mathrm{O}_{3^{-}}$ $0.27 \mathrm{PbTiO}_{3}$ single crystals were grown using the vertical Bridgman technique. ${ }^{13}$ Platelet-like $(\sim 5 \times 5 \times 0.6 \mathrm{~mm})$ samples with [001], [110], and [111] surface normal orientations were cut from very close positions in the as-grown crystal boule to ensure compositional consistency. The samples were then subjected to a series of polishings with the final polishing agent being an $\mathrm{Al}_{2} \mathrm{O}_{3}(\sim 50 \mathrm{~nm})$ aqueous suspension. Conductive silver paste (Ted Pella) was painted on the respective surfaces as a temporary electrode for poling the crystals under an external electric field of $10 \mathrm{kV} / \mathrm{cm}$. The painted 
TABLE I. Specifications of AFM probes used in this study. Note the calibrated force constants refer to the probes used for Fig. 7.

\begin{tabular}{lcccc}
\hline \hline & & & \multicolumn{2}{c}{ Force constant $(\mathrm{N} / \mathrm{m})$} \\
\cline { 4 - 5 } Probe & Tip radius (nm) & Tip coating (nm) & Nominal & Calibrated \\
\hline AC240TM & $28 \pm 10$ & $\operatorname{Ti} / \operatorname{Pt}(5 / 20)$ & 2 & 1.8 \\
PPP-NCHPt & $30 \pm 10$ & $\mathrm{Cr} / \mathrm{PtIr}(5 / 25)$ & 42 & 33.7 \\
\hline \hline
\end{tabular}

electrodes were then removed with acetone. The same silver paste was used to glue the samples onto metal AFM sample mounting discs, as well as to serve as a bottom grounded electrode.

PFM investigations were performed under ambient conditions on a commercial AFM system (Cypher, Asylum Research), which has integrated signal generator and lock-in amplifier (LiA) modules. Two types of conductive silicon probes, Olympus AC240TM and Nanosensors PPP-NCHPt, were used. Table I lists the specifications of the probes used and the detailed calibration in which the effective force constants of the probes were determined using the thermal noise method. The imaging contact set-points used were around $100 \mathrm{nN}$ and $500 \mathrm{nN}$ with the soft and stiff probes, respectively. For PFM domain imaging, we used off contactresonance ac probing signals (typically $10 \mathrm{kHz}$, amplitude $\left.V_{\mathrm{AC}}=1-5 \mathrm{~V}\right)$ to excite surface oscillations. These signal levels had no noticeable modifying effects on the pristine domain structures, as evidenced by consistent images obtained from repetitive scans. Both vertical (VPFM) and lateral (LPFM) signals were acquired on the crystals in each orientation by routing the corresponding detector channels to the LiA; generally, LPFM images were attained by scanning small regions and at a slow rate of $0.5 \mathrm{~Hz}$. To enhance signal-to-noise ratios and minimize perturbation for local $P R$ loop measurements, dual ac signals $\left(V_{\mathrm{AC}}=1 \mathrm{~V}\right.$, near $280 \mathrm{kHz}$ with AC240TM probes) were superimposed on a $0.5 \mathrm{~Hz}$ triangular staircase wave based on the dual ac resonance tracking (DART) technique. ${ }^{14}$ Both the writing and reading time were $25 \mathrm{~ms}$, and only the remnant-state data are presented. ${ }^{15}$ For local domain poling studies, an internal high voltage amplifier (HVA) was used to provide voltages greater than $10 \mathrm{~V}$ and a digital-to-analog (D/A) converter was used to generate pulses with various widths and a rising time of $7 \mu$ s (when coupled with the HVA).

\section{RESULTS AND DISCUSSIONS}

\section{A. Surface domain structures}

Figures 1(a) and 1(c) show the surface morphology and PFM phase image, respectively, of the pristine [110]-PIMNT single crystals. An apparent domain contrast can be observed in the former after polishing (Fig. 1(a)) as the downwards polarized domains are $1-2 \mathrm{~nm}$ higher, i.e., less abraded, than those polarized upwards. It seems that here a mechanochemical polishing effect produces the domain contrast. ${ }^{16}$ Figs. 1(b) and 1(d) are PFM phase images of the [001]- and [111]-PIMNT single crystals, respectively. Note that only phase images are presented throughout this paper, since the associated amplitude images usually show an ideal contrast where antiparallel domains have similar amplitudes and domain walls have near-zero values. ${ }^{12}$ From Figs. 1(b) and 1(d), both [001] and [111] crystals exhibit a labyrinth-like surface domain pattern. The domains are of $\sim 150 \mathrm{~nm}$ in characteristic widths while their orientations appear quite random. It is noteworthy that the piezoresponse on the (111) face is much weaker than on the (001) as well as (110) faces,
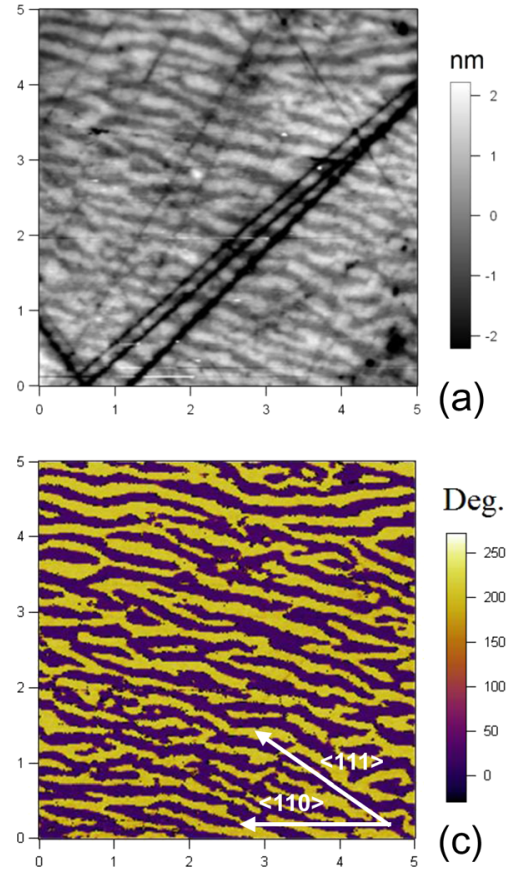

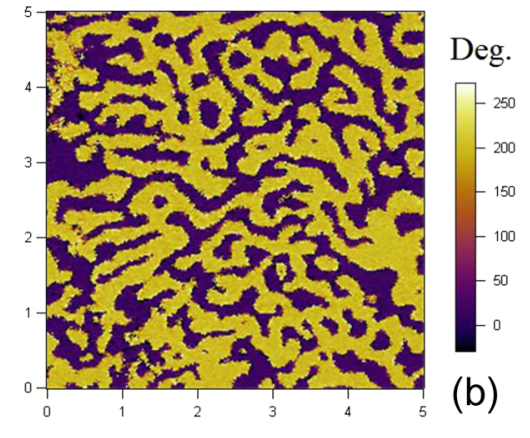

(b)

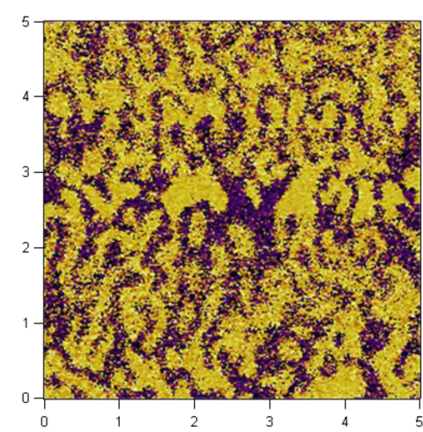

(d)
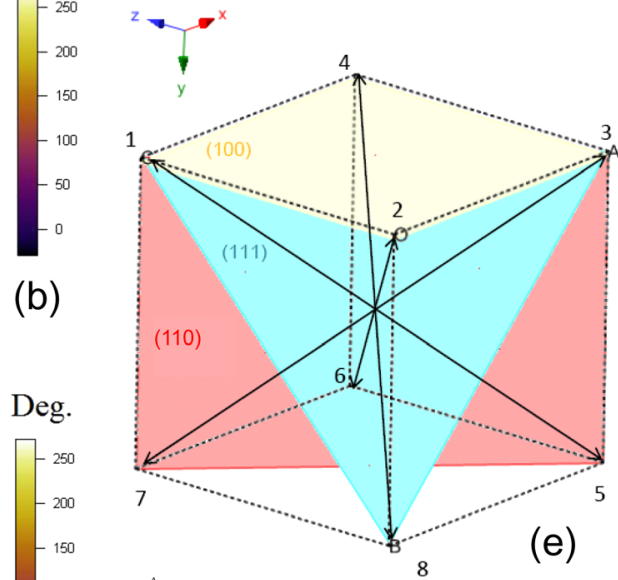

tip

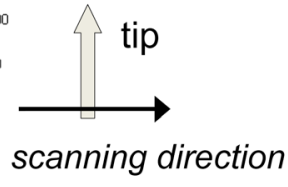

FIG. 1. (a) Surface morphology of [110]-PIMNT single crystals and PFM phase images of (b) [001]-, (c) [110]-, and (d) [111]-PIMNT single crystals. The scan sizes of all the images are $5 \times 5 \mu \mathrm{m}^{2}$, AC240TM probes used. The scanning direction (orthogonal to cantilevers) is also indicated. (e) Schematic of the crystal faces and the eight polarization vectors of a rhombohedral symmetry: $\boldsymbol{P}_{15}, \boldsymbol{P}_{51}, \boldsymbol{P}_{26}, \boldsymbol{P}_{62}, \boldsymbol{P}_{37}, \boldsymbol{P}_{73}, \boldsymbol{P}_{48}$, and $\boldsymbol{P}_{84}$. 
Their effective piezoelectric coefficients $\left(d_{33 \text {,eff }}\right)$ are estimated to be around $3 \mathrm{pm} / \mathrm{V}, 20 \mathrm{pm} / \mathrm{V}$, and $40 \mathrm{pm} / \mathrm{V}$, respectively. These estimates actually coincide well with the orientation dependence of the macroscopic $d_{33}$ reported previously. ${ }^{17}$ For this PIMNT composition, $d_{33}$ is $\sim 70 \mathrm{pC} / \mathrm{N}$ for [111] compared to near $2000 \mathrm{pC} / \mathrm{N}$ for [001] and [110] orientations. Although the PFM signal formation mechanism is very involved, it is apparent that the anisotropy of piezoelectricity in RSFCs still holds on a very local, e.g., several nanometer, scale.

By contrast, the domain pattern on the (110) face (see Fig. 1(c)) is more ordered and exhibits less curvature. Stripelike domains propagating over several microns without branching can be easily observed and, remarkably, show clear preferential orientation along $\langle 110\rangle$ as confirmed by equivalent phase images taken over many regions. An autocorrelation and fast Fourier transform (FFT) analysis performed on these domain patterns ${ }^{12}$ also verify the onedirection translational order on the (110) face as opposed to a seemingly in-plane isotropic feature shared by the other two faces [the (001) face is probably of a lower, 4-fold rotation symmetry in nature]. In addition, it should be noticed that the characteristic domain widths are similar on all faces.

The relationship between crystal orientation and the eight possible local spontaneous polarization, $\boldsymbol{P}$, vectors is illustrated in Fig. 1(e), drawn relative to a pseudo-cubic unit cell. Note that the domain structures discussed so far are all vertical, i.e., for each crystal orientation, only out-of-plane polarization components are measured. Therefore, all eight possible $\boldsymbol{P}$ vectors contribute equally to the VPFM signal for the [001] crystal, only four of the $\boldsymbol{P}$ vectors $\left(\boldsymbol{P}_{26}, \boldsymbol{P}_{62}, \boldsymbol{P}_{48}\right.$, and $\boldsymbol{P}_{84}$ ) for the [110] crystal and largely only two of the $\boldsymbol{P}$ vectors $\left(\boldsymbol{P}_{26}\right.$ and $\left.\boldsymbol{P}_{62}\right)$ for the [111] crystal. In the last case, the other six $\boldsymbol{P}$ vectors only have one-third vertical components. According to the above analysis, one can infer immediately that the four purely in-plane $\boldsymbol{P}$ vectors $\left(\boldsymbol{P}_{15}, \boldsymbol{P}_{51}, \boldsymbol{P}_{37}\right.$, and $\left.\boldsymbol{P}_{73}\right)$ are absent for the surface domains of the [110] crystal, since its VPFM result has no in-between piezoresponse other than that of antiparallel domains. Here the buckling effect, i.e., the contribution of the in-plane, parallel-to-thecantilever $\boldsymbol{P}$ components to the vertical signals, can be neglected as the PFM images after a $90^{\circ}$ sample rotation show essentially the same contrast. ${ }^{18}$ Nevertheless, we do not exclude the possible existence of large size ferroelastic twin domains beyond the lateral range of our PFM/AFM images $(\max .30 \mu \mathrm{m}){ }^{19,20}$

To obtain more information about the domain alignment, LPFM and VPFM images were taken at the same locations, as shown in Fig. 2 (the drift between them is negligible as judged by corresponding topographic images). For the [001] crystal (Figs. 2(a) and 2(e)), the lateral domain pattern is quite similar to the vertical pattern, mainly differing only slightly in domain widths and boundaries for which the uncertainties of LPFM, such as the shear strain effect, should be factored in. ${ }^{21}$ This clear resemblance indicates that most antiparallel domains in VPFM are arguably nonferroelastic (or $180^{\circ}$ domains, e.g., $\boldsymbol{P}_{51}$ and $\boldsymbol{P}_{15}$ ), although strictly speaking we need another orthogonal LPFM image to rule out the possibility of $109^{\circ}$ domains (e.g., $\boldsymbol{P}_{51}$ and $\left.\boldsymbol{P}_{48}\right)$. On the other hand, we do observe ferroelastic domains that can be firmly asserted from only two piezoresponse vectors, such as the region marked on Fig. 2(e). Such domains are ubiquitous on the (001) face, usually occurring as joints between two domain segments. A line poling experiment performed at the same location confirms the occurrence of ferroelastic domains. As expected, the VPFM image after poling shows two large antiparallel domains separated by a quite straight domain wall (Fig. 2(b)). The corresponding LPFM image also shows, roughly, two distinct sections. However, within each section one can still see minor irregularly shaped antiparallel domains regions not apparent in the pristine state (Figs. 2(e) and 2(f)). These "residual" LPFM
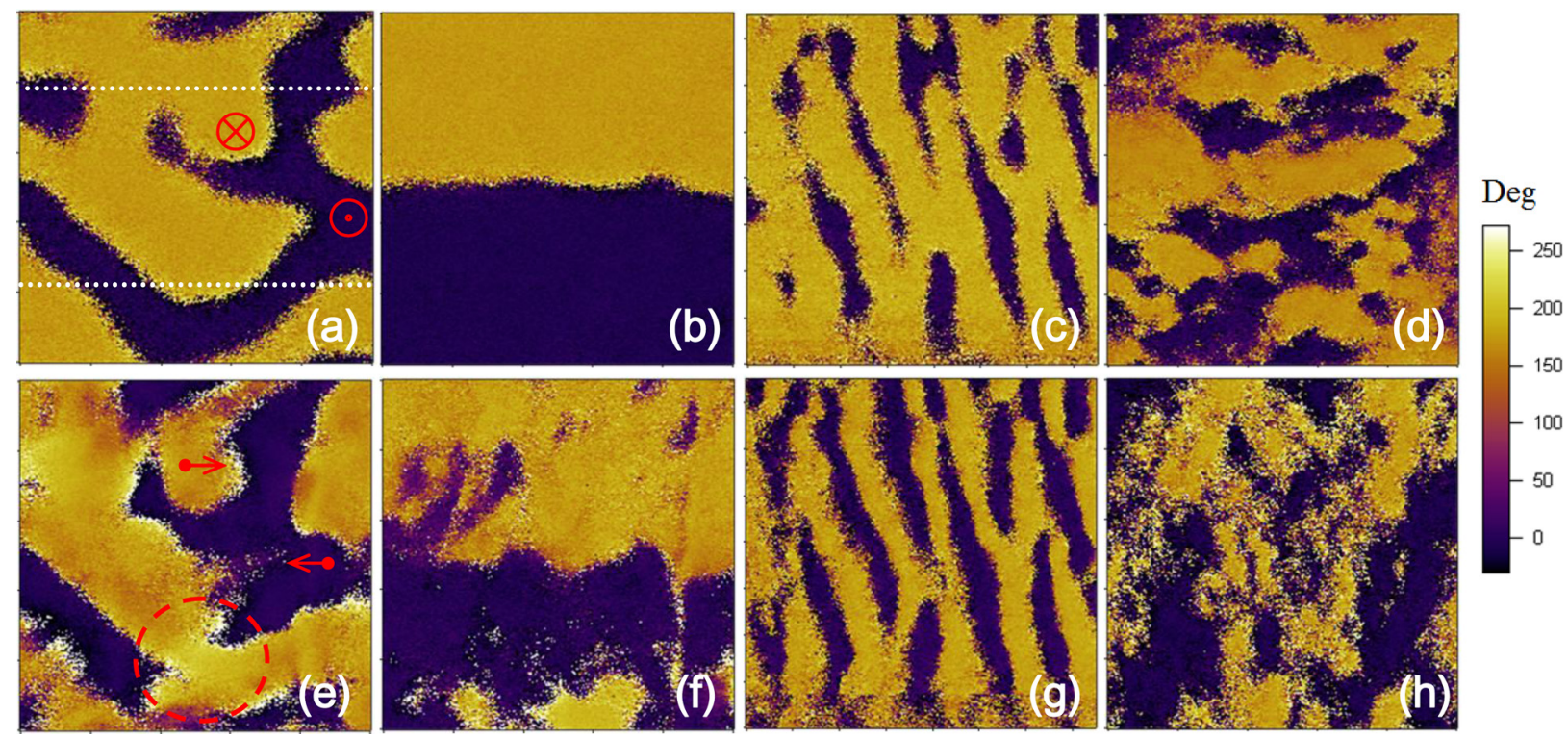

FIG. 2. (a)-(d) VPFM and (e)-(h) LPFM phases images of (a) and (e) [001]-, (b) and (f) after line poling, [001]-, (c) and (g) [110]-, and (d) and (h) [111]PIMNT single crystals. The dotted lines on (a) show the poling lines along which the tip was dragged at a $200 \mathrm{~nm} / \mathrm{s}$ speed with $20 \mathrm{~V}$ (upper) and - $20 \mathrm{~V}$ (lower) applied. The arrows represent the polarization directions. The scan sizes are $1 \times 1 \mu \mathrm{m}^{2}$ except $1.5 \times 1.5 \mu \mathrm{m}^{2}$ for the [110] crystal, PPP-NCHPt probes used. 
domains strongly suggest that local polarization switching for RFSCs under tip biases is not a straightforward $180^{\circ}$ dipole flip-over process. It may instead involve a nonuniform dipole reorientation process that is of a ferroelastic nature.

By comparison with the (001) face images, the LPFM and VPFM images of the (110) face show an even higher resemblance (cf., Fig. 2(c) with Fig. 2(g)). All domains appear to be of $180^{\circ}$ type, in agreement with the absence of purely in-plane $\boldsymbol{P}$ vectors inferred above. By contrast, there is no clear correlation between the LPFM and VPFM images for the (111) face images (cf., Figs. 2(d) and 2(h)). The LPFM domains are highly diffuse and featureless, suggesting that various types of $\boldsymbol{P}$ vectors mix randomly in terms of their lateral components on the (111) face.

While domain structures have been observed on all three crystal faces using VPFM and LPFM, it is still a challenging task to spatially correlate them from the classical ferroelectric domain configuration point of view where the elastic conformation and electrostatic energy minimization conditions determine an equilibrium state. ${ }^{22}$ In normal displacive ferroelectrics, well-defined polar dipoles down to the single unit cell level are long-range correlated. In the case of relaxor ferroelectrics, however, the minimum polar units are a few nm sized PNRs, which can be both dynamic and interactive. Labyrinth-like domains, as frequently observed in a variety of RFSCs, are considered a mesoscopic hallmark of the existence of disordered PNRs. A recent paper of Kholkin et al. ${ }^{9}$ has shown that broken symmetry at the surface is the key to the formation of surface domains by altering the interactions (elastic, electrostatic, and other) between PNRs therein. Here, our results show explicitly the orientation dependence of surface domain patterning for PIMNT crystals.
The results appear to agree with the grain dependence Kholkin et al. ${ }^{9}$ reported for relaxor $\mathrm{Pb}_{0.905} \mathrm{La}_{0.095} \mathrm{Zr}_{0.65} \mathrm{Ti}_{0.35} \mathrm{O}_{3}$ polycrystalline samples. ${ }^{9}$ Given the microscopic picture drawn from reciprocal space that $\langle 110\rangle$ PNRs $\left(\boldsymbol{P}_{\mathrm{PNR}}\right)$ are embedded into $\langle 111\rangle$-polarized ferroelectric matrices, ${ }^{23}$ we believe the orientation dependence to be the result of a PNRmodulation effect. On the (110) face, two in-plane $\boldsymbol{P}_{\mathrm{PNR}}$ 's are parallel to the domain walls favored by the rhombohedral matrix, thus allowing their long range propagation along the $\langle 110\rangle$ direction. Otherwise, for example, on the (001) face there are four $\langle 110\rangle$-type $\boldsymbol{P}_{\mathrm{PNR}}$ in plane, all of which reside in each single domain with equal likelihood thus frustrating a long-range ordered assembly of the domains. For all cases, the domain width reflects the modulation strength of the PNRs and seems less affected by crystal orientation.

\section{B. Piezoresponse hysteresis loops}

For the [001]-PIMNT single crystals, local piezoresponse hysteresis loops were acquired on a grid of $30 \times 30$ pixels evenly distributed over a $0.8 \times 0.8 \mu \mathrm{m}^{2}$ region. The characteristic parameters of each $P R$ loop are calculated and then constructed into a spatially correlated image. The method is known as switching spectroscopy (SS-PFM) mapping. Fig. 3 shows the resultant switching maps including (a) a topography image, (b) the corresponding coercive voltage map, defined as $\left[V_{\mathrm{c}}(+)-V_{\mathrm{c}}(-)\right] / 2\left[V_{\mathrm{c}}\right.$ : the voltage where $P R$ measures as zero; see Fig. 3(d)], and (c) the built-in voltage map, defined as $\left[V_{\mathrm{c}}(+)+V_{\mathrm{c}}(-)\right] / 2$. The contour of the labyrinth domain pattern, obtained from a previous VPFM image of the same area, is overlaid on the topographic image in Fig. 3(a). In general, all the hysteresis loops show weak position dependence with the majority of coercive voltages

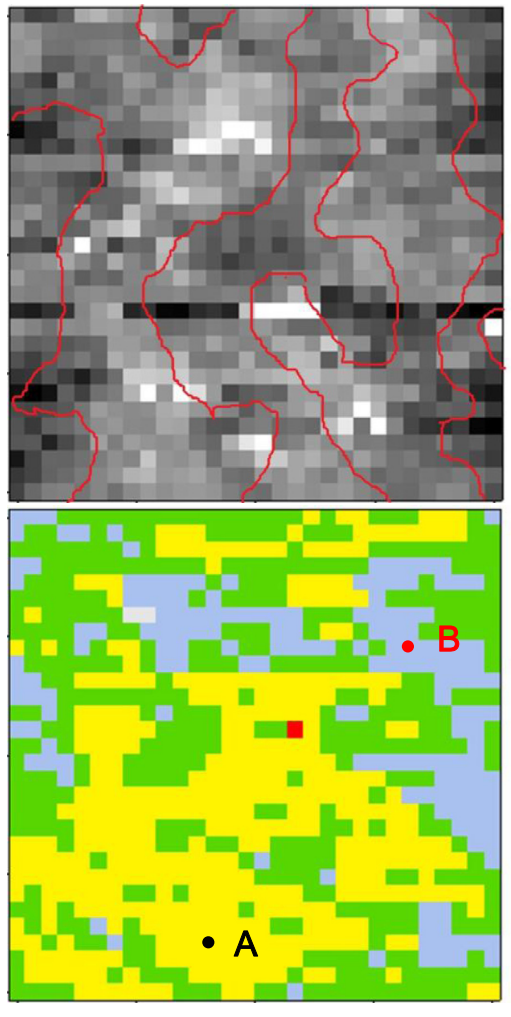

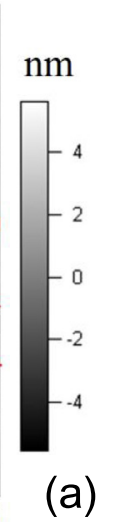
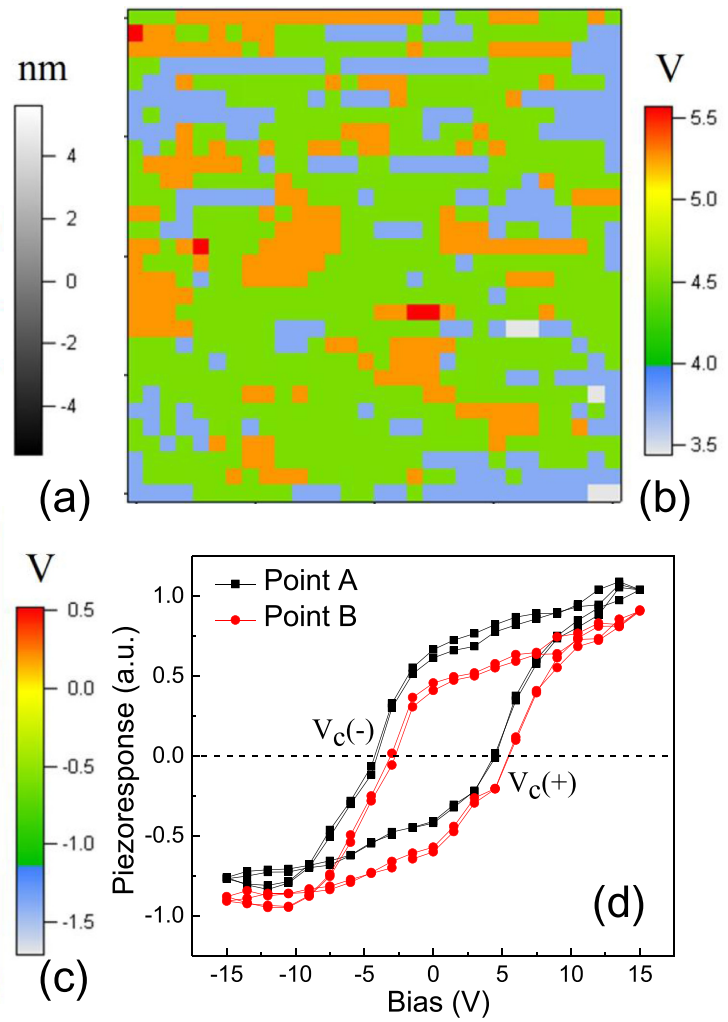

FIG. 3. (a) topography image, (b) coercive voltage map, and (c) built-in voltage map reconstructed from the switching force curve map $(30 \times 30$ pixels $)$ of a [001]PIMNT crystal. (d) Typical hysteresis loops measured on the locations as marked on (c). The domain pattern is delineated on (a). The image size is $0.8 \times 0.8 \mu \mathrm{m}^{2}$, AC240TM probes were used. 
being $\sim 4.5 \mathrm{~V}$ (Fig. 3(b)). Despite a larger macroscopic coercivity that PIMNT crystals show in the polarization hysteresis $(P-E)$ loop measurement, the coercive voltage level is similar to that measured on $\mathrm{Pb}\left(\mathrm{Mg}_{1 / 3} \mathrm{Nb}_{2 / 3}\right) \mathrm{O}_{3}-x \mathrm{PbTiO}_{3}{ }^{7,8}$ This further verifies that in RSFCs the random field and random bond defects, as Rodriguez et al. ${ }^{7}$ argues, can significantly promote nucleation during local domain switching. The $P R$ loops tend to be symmetric along the voltage axis, resulting in small measured built-in voltages (Fig. 3(c)). Nevertheless, there are several well separated regions showing large built-in voltages up to $\sim 1.5 \mathrm{~V}$. Two representative loops (Fig. 3(d)) collected from different areas exhibit nearly the same shapes and magnitudes, with one of them being offset horizontally, as a result of the underlying built-in fields.

It has been confirmed that the kinetics of $P R$ hysteresis loops are very sensitive to local charge fields seen by the switching domains. ${ }^{3}$ In RFSCs, the $B$-sites of the average perovskite structure are occupied by multiple ions, e.g., $\mathrm{Mg}^{2+}, \mathrm{Nb}^{5+}$, etc. An inhomogeneous distribution and/or local ordering of these aliovalent ions can give rise to local charge fields. Besides, structural defects such as oxygen vacancies and charged domain walls can also contribute to the space charge fields. The distribution of those built-in fields, on the other hand, shows little spatial correlation with the pristine domain pattern (see Fig. 3(a)), confirming that the formation of labyrinth-like domains is independent of the fluctuations of local built-in fields. ${ }^{7,8}$

Fig. 4 shows representative $P R$ loops obtained from [110]-oriented and [111]-oriented PIMNT crystals. The loops for the [110] crystals have similar shapes and characteristic parameters, e.g., coercive voltages, to those obtained from the [001] oriented crystal. By comparison, it is difficult to measure a well-formed $P R$ loop at random positions on the [111] crystals as found for SS-PFM mapping while high voltages are necessary to obtain saturated loops. The coercive voltage for the [111] crystal is around 12-16 V (estimated from $\sim 30$ loops acquired at different locations), markedly higher than the corresponding values for the [001] and [110] crystals. Besides, the [111] $P R$ loops are found to vary greatly in appearance depending on location. In Fig. 4(b), for example, the $P R$ loops at point 1 are symmetric, while those at point 2 are remarkably asymmetric along both the voltage and $P R$ axis, indicative of a built-in field and non-switchable (frozen) polarization. The frozen polarization is attributed to the strong pinning effect from the built-in field. ${ }^{24}$ Note here that the $P R$ magnitudes at the two points may be not directly comparable for lack of $Q$ (resonance quality factor) information. ${ }^{14}$ Even without the SS-PFM maps, it appears that the space charge fields in the case of the [111] oriented crystals have larger strengths as well as fluctuations. This is somewhat unexpected, particularly in the sense that all three orientations show very close $(\sim 5 \mathrm{kV} /$ $\mathrm{cm})$ coercive fields from the measured $P$-E loops. This may imply that some charged defects are correlated along [111], the polar axis, on a local scale (presumably, a few hundred $\mathrm{nm}$ ). Further SS-PFM mapping would be necessary to reveal their relation to the observed domain patterns.

\section{Local domain poling studies}

In light of the above domain imaging and $P R$ hysteresis loop results, we have carried out domain poling studies mainly on the [001] oriented PIMNT crystal, using an externally downwards poled sample, as a result of the relative ease of being poled and detected. The main parameters for the poling experiments are specified below. The domain sizes are measured from the PFM phase images. We use the effective diameter $D_{E}$, as derived from the measured area. The uncertainty of such a measurement is around two pixels in size.

We have previously reported ${ }^{25}$ abnormal domain switching (back-switching) behavior that occurs when the bias (either positive or negative) applied to tips is high enough to induce charge injection. To avoid this effect, the tip voltage is retained until the tip is taken off the sample. This nullifies the grounded boundary condition for the nearsurface regions filled with space charges (injected carriers). As a consequence, the back-switching phenomenon can be effectively eliminated. ${ }^{26}$ As shown in Fig. 5, uniform nearround domains with an upwards polarization direction can be produced under a series of negative tip voltages by this procedure. The effective diameter, $D_{E}$, of these domains follows a highly linear dependence on the tip voltage. Experimentally, this dependence has been confirmed for a variety of ferroelectric materials, such as bulk $\mathrm{LiNbO}_{3}$ and $\mathrm{BaTiO}_{3}$ as well as $\mathrm{Pb}(\mathrm{Zr}, \mathrm{Ti}) \mathrm{O}_{3}$ thin films. ${ }^{27,28}$ It is well accepted that the tip-induced polarization switching in PFM complies with a general model of nucleation and lateral domain wall motion, which can be expressed by the following formula: ${ }^{29}$

$$
D_{E} \propto U \ln U^{-1} v_{\infty} \delta \tau,
$$
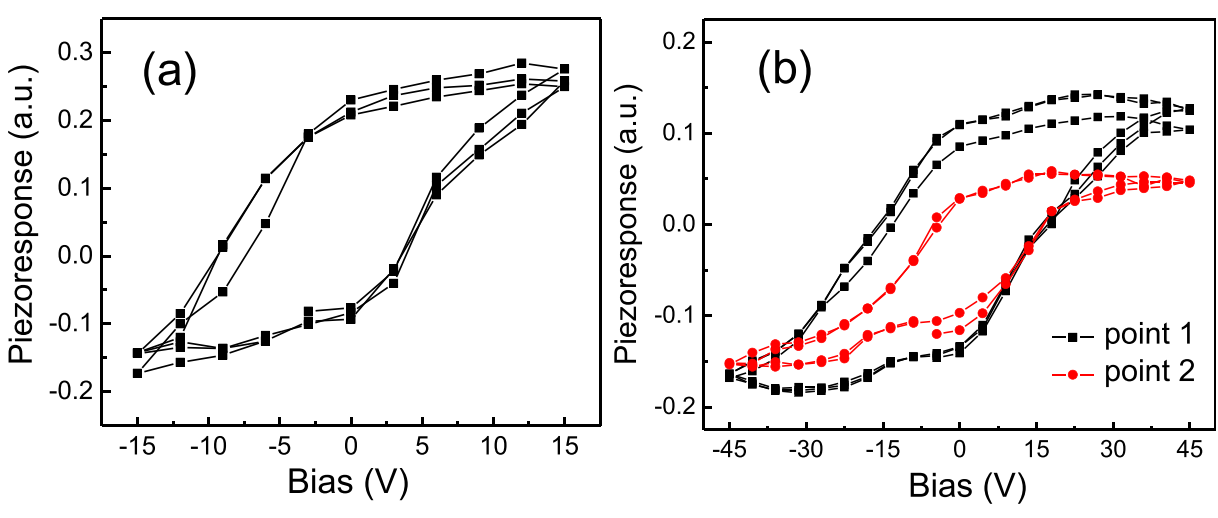

FIG. 4. Typical piezoresponse hysteresis loops measured on the (a) [110]- and (b) [111]-PIMNT single crystals. AC240TM probes were used. 


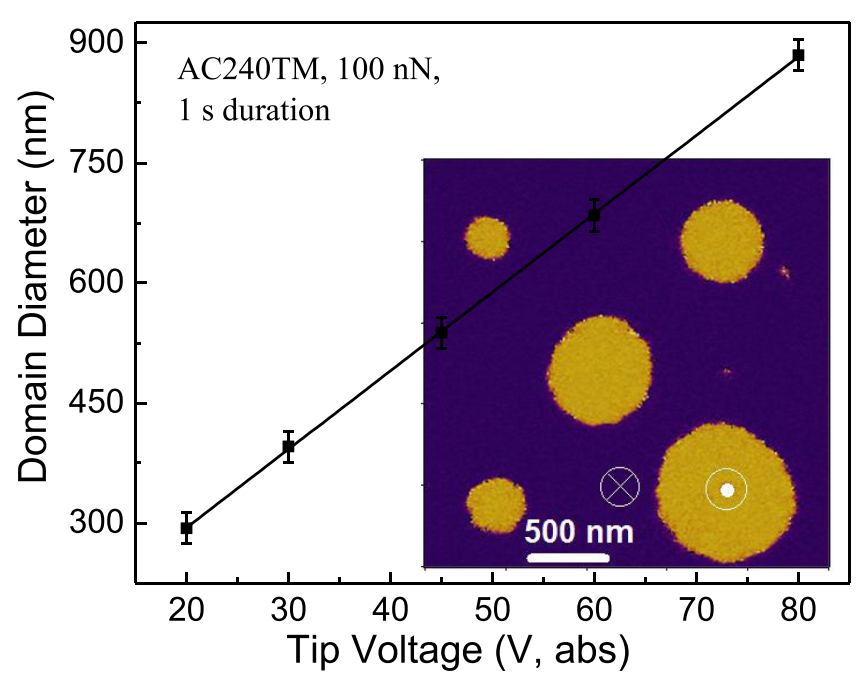

FIG. 5. Domain diameter as a function of tip voltage applied for $1 \mathrm{~s}$ durations, using an AC240TM probe with $100 \mathrm{nN}$ loading forces. Inset shows the PFM phase images, with the arrows representing the polarization directions.

where $U$ and $\tau$ represent the tip voltage and its duration, respectively. $\nu_{\infty}$ and $\delta$ are parameters defining the electric field $E$-dependent domain wall velocity $v_{\mathrm{dw}}$ as follows: $v_{d w}$ $=v_{\infty} \exp (-\delta / E)$. Here, the field $E$ is given by a single point charge model. Formula (1) gives the linear relationship between $D_{E}$ and $U$ using a first order approximation.

Fig. 6 shows the dependence of $D_{E}$ on the duration of the applied tip voltages. For pulses with width $w \geq 1 \mathrm{~s}$, the same procedure as described above was used. Due to difficulties in synchronizing tip motions with the application of voltage, pulses with $w<1 \mathrm{~s}$ were applied during $1 \mathrm{~s}$ tipsample engagements. Both AC240TM and PPP-NCHPt probes were used with corresponding loading forces, resulting in an interesting comparison. The domains produced with an AC240TM probe experienced back-switching for

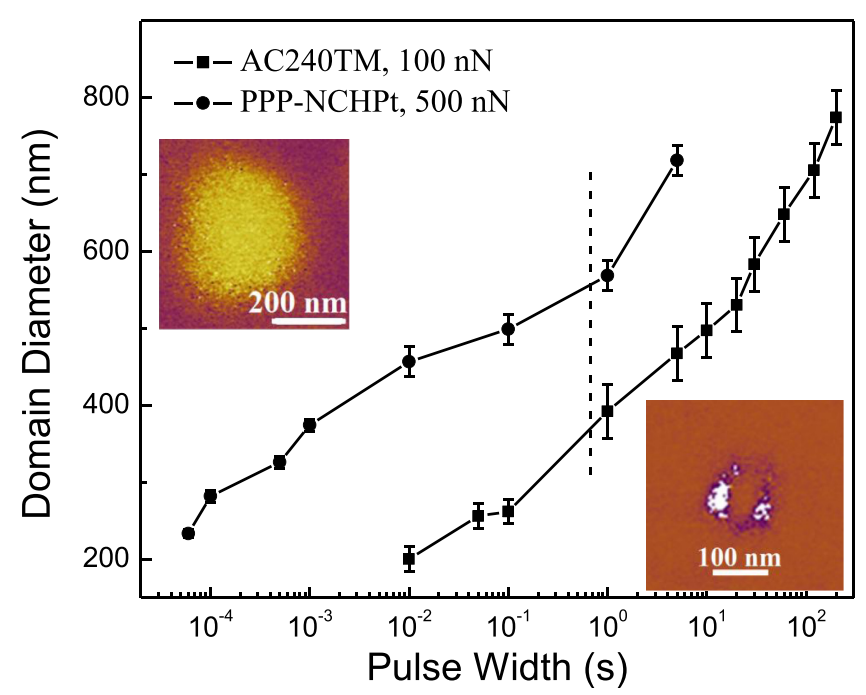

FIG. 6. Domain diameter as a function of pulse width under a tip voltage of $-30 \mathrm{~V}$. Insets show the domains created by $0.5 \mathrm{~ms}$ width pulses with AC240TM (right) and PPP-NCHPt (left) probes. The back-switched areas are included for calculating the domains that experienced back-switching. The dashed line indicates different methods used for the two regions. pulses $w<1 \mathrm{~s}$, as the tip was grounded shortly before being withdrawn. Especially, when $w<10 \mathrm{~ms}$, the normally (upwards) poled domains can be largely back-switched and even entirely disappear thereby (see e.g., the right inset in Fig. 6). By contrast, all pulses (min. $60 \mu \mathrm{s}$ ) applied via a PPP-NCHPt probe produce well-defined upwards polarized domains without noticeable back-switching (see the left inset in Fig. 6). Note that the domains achieved with the PPPNCHPt probe are significantly larger than those achieved with the AC240TM probe. On the other hand, both sets of $D_{E}$ data exhibit a satisfactory logarithmic dependence on pulse duration $\tau$, as dictated by Formula (1). Similar slopes obtained from the $D_{E^{-}} \log (\tau)$ plots indicate that the domain wall velocity $v_{d w}$ is similar but slightly different in the two cases.

Usually the domain growth kinetics under a tip bias is extrinsically affected by the non-homogenous electric field produced via the probe. Solving for the distribution of this field is nontrivial in real situations, because it is not only an intrinsic coupled problem involving both mechanical stress and electrostatic fields but is also affected by surface reactivity $^{30}$ as well as the charge injection effect. In the last result, both types of probes used have the same nominal tip radius $\sim 30 \mathrm{~nm}$. Therefore, only the one controlled parameter, the loading force $F$, was different. A subsequent experiment was conducted by varying $F$ using each probe. Note that the large potential difference between the tip $(30 \mathrm{~V})$ and sample surface leads to an additional indentation force, which was not calibrated for here. As shown in Fig. 7, $D_{E}$ in general increases with $F$ in the low force region, particularly evident for the AC240TM probe, but tends to become flatter for the PPP-NCHPt probe, suggesting a saturation effect for high loading forces. The former $D_{E}$ versus $F$ curve can be reasonably well fitted by a power function of the form $D_{E} \propto F^{0.35}$. Interestingly, in the pure elastic Hertzian contact model, ${ }^{3}$ the contact radius $R_{\mathrm{C}}$ scales with loading force in a similar manner, $R_{\mathrm{C}} \propto F^{1 / 3}$. Note that the contact radius, $R_{\mathrm{C}}$ is far smaller than the domain size $D_{E}$, being only a few $\mathrm{nm}$. The scaling similarity may be

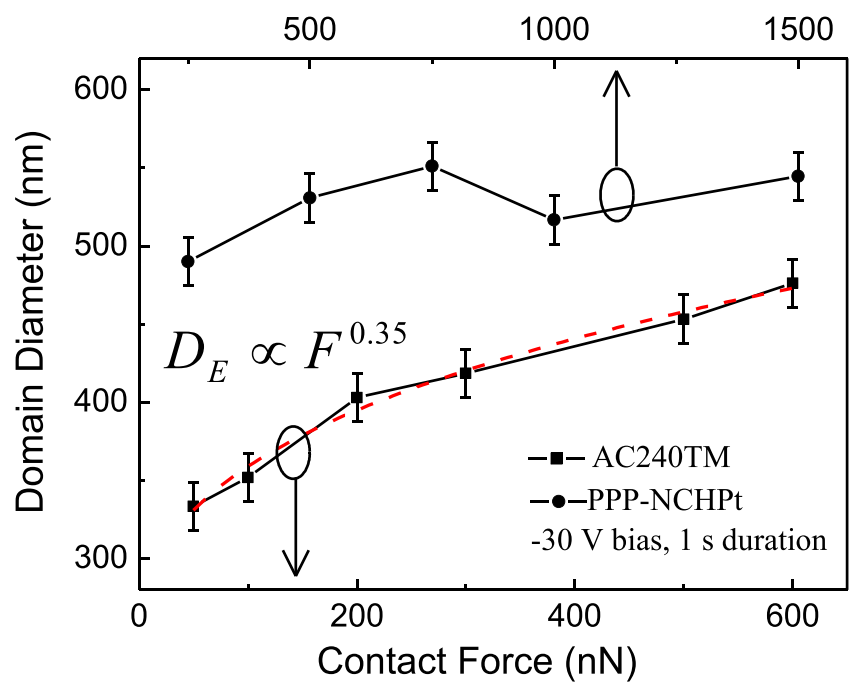

FIG. 7. Domain diameters as a function of loading force under $-30 \mathrm{~V}$ tip voltages for $1 \mathrm{~s}$ durations. 
rationalized by the presumption that the attenuation factor $\gamma$ (defined by $\left.V_{\text {surface }}=\gamma V_{\text {tip }}\right)^{3}$ of the absorption layers, which inevitably resides on the sample surface in ambient conditions ${ }^{31}$ can be modified by loading force. In other words, the effective field within the sample does change with the force exerted on the tip. This may account largely for the different sizes (Fig. 6) of the domains poled using the two types of probes.

Finally, the problem still remains as to why backswitching is not observed for domains poled with the PPPNCHPt probe. One possible explanation is that the injected charges are so few that the resultant space charge field is not strong enough to invert the domains in this case. We speculate that the work functions $\Phi_{\mathrm{m}}$ of the two tips are different perhaps simply owing to the dissimilarity in the coating materials, giving rise to different Schottky barrier heights $\Phi_{\mathrm{b}}$ between the tip-sample junction. ${ }^{32}$ Assuming that the crystal is electrically homogenous, the amount of carriers injected is proportional to the reverse current. Then under the same reverse bias, from the Schottky emission equation, ${ }^{32}$ we see that the reverse current scales logarithmically with the barrier height $\Phi_{\mathrm{b}}$; only a small difference of $\Delta \Phi_{\mathrm{b}}=0.12 \mathrm{eV}$ at room temperature can result in an over 100-fold reduction in the amount of injected carriers. Further direct electric transport measurement would provide more evidence for this aspect, currently impeded by the high bulk resistivity of the sample.

\section{SUMMARY}

In summary, we have systematically investigated the domain structures and local switching characteristics of PIMNT single crystals with [001], [110], and [111] orientations using PFM imaging and spectroscopy techniques. The labyrinth domain patterns, observed on both (001) and (111) surfaces, are quite consistent with those reported for other RFSC systems. A careful analysis of VPFM and LPFM results suggests the possibility of polarization vector alignment on each crystal surface, and also shows a non-deterministic reversal of the lateral polarization components during poling for the [001] oriented crystal. The local piezoresponse switching loops show that larger coercive voltages are required for [111] oriented crystals than for the other orientations, indicative of a higher built-in field that strongly pins local domain nucleation and growth. Finally, the tip-induced domain growth kinetics on a poled [001] crystal is studied as a function of several experimental parameters. The resultant domain size has linear and logarithmic dependence on the magnitude and duration of tip bias, respectively. The loading force and tip chemical attributes are also found to play an important role in determining local electrical fields and potential charge transport phenomena, consequently affecting the domains produced.

\section{ACKNOWLEDGMENTS}

Q.L., Y.L., and R.L.W. appreciate the support of the Australian Research Council (ARC) in the form of a Discovery Grant. Y.L. also appreciates support from the ARC Future Fellowships program.

${ }^{1}$ Scanning Probe Microscopy: Electrical and Electromechanical Phenomena at the Nanoscale, edited by S. V. Kalinin and A. Gruverman (Springer, New York, 2007).

${ }^{2}$ S. V. Kalinin and D. A. Bonnell, Phys. Rev. B 65, 125408 (2002).

${ }^{3}$ S. V. Kalinin, A. N. Morozovska, L. Q. Chen, and B. J. Rodriguez, Rep. Prog. Phys. 73, 056502 (2011).

${ }^{4}$ A. Baji, Y. W. Ma, Q. Li, and Y. Liu, Compos. Sci. Technol. 71, 1435 (2011). ${ }^{5}$ M. Abplanalp, D. Barošová, P. Bridenbaugh, J. Erhart, J. Fousek, P. Günter, J. Nosek, and M. Šulc, J. Appl. Phys. 91, 3797 (2002).

${ }^{6}$ V. V. Shvartsman and A. L. Kholkin, Phys. Rev. B 69, 014102 (2004).

${ }^{7}$ B. J. Rodriguez, S. Jesse, A. N. Morozovska, S. V. Svechnikov, D. A. Kiselev, A. L. Kholkin, A. A. Bokov, Z.-G. Ye, and S. V. Kalinin, J. Appl. Phys. 108, 042006 (2010).

${ }^{8}$ S. V. Kalinin, B. J. Rodriguez, J. D. Budai, S. Jesse, and A. N. Morozovska, Phys. Rev. B 81, 064107 (2010).

${ }^{9}$ A. Kholkin, A. N. Morozovska, D. Kiselev, I. Bdikin, B. Rodriguez, P. Wu, A. Bokov, Z.-G. Ye, B. Dkhil, L. Chen, M. Kosec, and S. V. Kalinin, Adv. Funct. Mater. 21, 1977 (2011).

${ }^{10}$ A. A. Bokov and Z.-G. Ye, J. Mater. Sci. 41, 31 (2006).

${ }^{11}$ L. V. Simagina, E. D. Mishina, S. V. Semin, N. A. Ilyin, T. R. Volk, R. V. Gainutdinov, and L. I. Ivleva, J. Appl. Phys. 110, 052015 (2011).

${ }^{12}$ See supplementary material at http://dx.doi.org/10.1063/1.4745979 for the electron diffraction results and the detailed imaging parameters, PFM amplitude images and autocorrelation/FFT analysis for Figs. 1 and 2.

${ }^{13}$ X. Wang, Z. Xu, Z. Li, F. Li, H. Chen, and S. Fan, Ferroelectrics 401, 173 (2010).

${ }^{14}$ B. J. Rodriguez, C. Callahan, S. V. Kalinin, and R. Proksch, Nanotechnology 18, 475504 (2007).

${ }^{15}$ S. Jesse, A. P. Baddorf, and S. V. Kalinin, Appl. Phys. Lett. 88, 062908 (2006).

${ }^{16}$ T. Scholz, B. Mihailova, G. A. Schneider, N. Pagels, J. Heck, T. Malcherek, R. P. Fernandes, V. Marinova, M. Gospodinov, and U. Bismayer, J. Appl. Phys. 106, 074108 (2009).

${ }^{17}$ S.-E. Park and T. R. Shrout, J. Appl. Phys. 82, 1804 (1997).

${ }^{18}$ E. Soergel, J. Phys. D: Appl. Phys. 44, 464003 (2011).

${ }^{19}$ J. Yao, Y. Yang, W. Ge, J. Li, and D. Viehland, J. Am. Ceram. Soc. 94, 2479 (2011).

${ }^{20}$ F. Bai, J. Li, and D. Viehland, Appl. Phys. Lett. 85, 2313 (2004).

${ }^{21}$ J. Guyonnet, H. Bea, F. Guy, S. Gariglio, F. Fusil, K. Bouzehouane, J.-M. Triscone, and P. Paruch, Appl. Phys. Lett. 95, 132902 (2009).

${ }^{22}$ A. K. Tagantsev, L. E. Cross, and J. Fousek, Domains in Ferroic Crystals and Thin Films (Springer, 2011).

${ }^{23}$ G. Xu, Z. Zhong, Y. Bing, Z.-G. Ye, and G. Shirane, Nature Mater. 5, 134 (2006).

${ }^{24}$ S. Jesse, B. J. Rodriguez, S. Choudhury, A. P. Baddorf, I. Vrejoiu, D. Hesse, M. Alexe, E. A. Eliseev, A. N. Morozovska, J. Zhang, L.-Q. Chen, and S. V. Kalinin, Nature Mater. 7, 209 (2008).

${ }^{25}$ Q. Li, Y. Liu, J. Schiemer, P. Smith, Z. Li, R. L. Withers, and Z. Xu, Appl. Phys. Lett. 98, 092908 (2011).

${ }^{26}$ M. Lilienblum and E. Soergel, J. Appl. Phys. 110, 052018 (2011).

${ }^{27}$ T. Tybell, P. Paruch, T. Giamarchi, and J.-M. Triscone, Phys. Rev. Lett. 89, 097601 (2002).

${ }^{28}$ B. J. Rodriguez, L. M. Eng, and A. Gruverman, Appl. Phys. Lett. 97, 042902 (2010).

${ }^{29}$ M. Lilienblum and E. Soergel, J. Appl. Phys. 110, 052012 (2011).

${ }^{30}$ S. V. Kalinin, S. Jesse, A. Tselev, A. P. Baddorf, and N. Balke, ACS Nano 5, 5683 (2011).

${ }^{31}$ V. Y. Shur, A. V. Ievlev, E. V. Nikolaeva, E. I. Shishkin, and M. M. Neradovskiy, J. Appl. Phys. 110, 052017 (2011).

${ }^{32}$ L. Pintilie and M. Alexe, J. Appl. Phys. 98, 124103 (2005). 